\title{
PRODUCCION INTEGRADA DE PAPA (Solanum Tuberosum L.): UN ANALISIS DE SENSIBILIDAD AGROECONÓMICA PARA EL SUDESTE BONAERENSE, ARGENTINA
}

\author{
J.A. Rodríguez ${ }^{1}$; E.M.M. Rodríguez ${ }^{1}$
}

Manuscrito recibido: $11 / 02 / 2014$

Aceptado: $25 / 11 / 2014$

Disponible en línea: Diciembre 2014

\section{Resumen}

La Producción Integrada (PI) es un Sistema Agrícola de Producción de alimentos que utiliza al máximo los recursos y los mecanismos de regulación naturales y asegura a largo plazo una agricultura viable. En el sudeste de Buenos Aires, Argentina, este sistema productivo ha sido plasmado en un Protocolo realizado por el INTA Balcarce para papa consumo. El objetivo de este trabajo es plantear diferentes escenarios cambiando variables críticas relacionadas con la producción agrícola -tales como los rendimientos y el precio de los productos- y estimar el beneficio neto contemplando diferentes tasas de descuento. Se estimaron los Costos de Producción Integrada de papa en el sudeste de la provincia de Buenos Aires. Se realizó un "Cash Flow" descontando los ingresos netos esperados y, finalmente, un análisis de sensibilidad con 27 escenarios posibles, donde se obtuvieron resultados negativos y positivos que oscilaron entre - 1,14 y 4,14 millones de pesos.

Palabras claves adicionales: Papa consumo, Producción Integrada, Costo de producción

1 Facultad de Ciencias Económicas y Sociales, Universidad Nacional de Mar del Plata, Argentina Email: july22r@yahoo.com.ar // july22r@hotmail.com 


\section{POTATO INTEGRATED PRODUCTION (Solanum tuberosum L.): A SENSITIVITY AGRO-ECONOMIC ANALYSIS FOR SOUTHEAST BONAERENSE, ARGENTINA}

\section{Summary}

Integrated Production (IP) is an Agricultural Food Production System that maximizes the use of resources and mechanisms of natural regulation and ensures long-term sustainable farming. In the southeast of Buenos Aires, Argentina this production system has been embodied in a Potato Protocol framework made by INTA, Balcarce. The aim of this paper is to discuss different scenarios changing critical variables related to agricultural production, such as the yields and the product sale price and estimate a "Cash Flow" considering different discount rates. Integrated Production Costs of fresh potato in southeastern Buenos Aires province were estimated in this paper. A "Cash Flow" was performed by discounting the expected net income and finally a sensitivity analysis with 27 scenarios obtained negative and positive results that ranged between - 1,14 and 4,14 million pesos.

Additional Keywords: Potato, Integrated Production, Production costs

\section{Introducción}

El surgimiento de consumidores con mayores exigencias de calidad y la ampliación de los mercados a nivel mundial, han llevado al aumento de las exigencias fitosanitarias y de inocuidad de los productos agrícolas. Frente a esto, se han comenzado a implementar diferentes procesos productivos que garanticen la calidad de los productos, respondan a las nuevas exigencias de los consumidores y, por último pero no menos importante, que sea factible su implementación.

La Producción Integrada (PI) es parte de este proceso de cambio. Es definida por la Organización Internacional de Lucha Biológica e Integrada como: "Un sistema agrícola de producción de 
alimentos que utiliza al máximo los recursos y los mecanismos de regulación naturales y asegura a largo plazo una agricultura viable. En ella los métodos biológicos, culturales, químicos y demás técnicas son cuidadosamente elegidos y equilibrados, teniendo en cuenta el medio ambiente, la rentabilidad y las exigencias sociales".

En años recientes se han creado una gran variedad de códigos, normas y reglamentos sobre Buenas Prácticas Agrícolas y Producción Integrada, con el objetivo de codificar las prácticas de una gran cantidad de productos a nivel de la explotación agrícola. El sudeste de la provincia de Buenos Aires no es la excepción ya que en el marco de un proyecto nacional de investigación en papa del INTA Balcarce, se ha desarrollado el Protocolo para la Producción Integrada de Papa Consumo en el Sudeste de la Provincia de Buenos Aires (Huarte et al, 2011).

Con la PI se busca obtener una papa de calidad culinaria superior -a través de la utilización de variedades alternativas de papa- a las más difundidas actualmente en el mercado como la variedad Spunta y un producto con un nivel menor de residuos agroquímicos. También se pretende obtener un mayor retorno económico de la actividad papera por mayor segmentación del mercado con un producto obtenido a través de prácticas productivas más sustentables e identificables por el consumidor.

La producción de papa es una modalidad productiva caracterizada por un elevado costo -en relación a otros cultivos propios de la región-, altamente dependiente de insumos importados y con precios que frecuentemente no llegan a cubrir los costos. Esta situación dificulta el abastecimiento de un producto de primera necesidad a un precio accesible al consumidor, y justifica explorar nuevas alternativas productivas (Huarte et al, 2011). En ese contexto, la Producción Integrada se constituye en una alternativa productiva amigable con el medio ambiente y las personas, además de ser una nueva alternativa comercial. 
El objetivo de este trabajo es comparar los Costos de Producción de Papa -Convencional e Integrada-bajo diferentes escenarios alterando variables críticas relacionadas con la producción agrícola como son los rendimientos y el precio de venta de los productos, y estimar el beneficio neto contemplando diferentes tasas de descuento.

\section{Materiales y métodos}

Se estimaron los Costos de Producción Integrada y Convencional de papa en el Sudeste de la Provincia de Buenos Aires, Argentina en un análisis de corto plazo.

Los cálculos se realizaron, principalmente, con los datos obtenidos a partir de entrevistas a informantes calificados - investigadores de la EEA INTA Balcarce, docentes de la Universidad Nacional de Mar del Plata, productores de papa, prestadores de servicios relacionados con la actividad, entre otros- y con información de fuentes secundarias -datos de organismos públicos, publicaciones científicas, etc.-

En el sector agrícola, cuando se hace referencia a los sacrificios de recursos necesarios para obtener un cultivo, se los relaciona con una extensión o superficie de tierra ubicada en una determinada zona o región (Remondino y Garino, 2003). En esta investigación ${ }^{2}$, se trabajó con una escala de producción determinada, debido a la importancia e incidencia que tiene en el cálculo de los costos de producción. Se consideró un lote de 80 hectáreas de papa (en el sudeste de la provincia de Buenos Aires, Argentina), siendo ese número recomendado por los informantes calificados ya que estiman que a partir de esa extensión de tierra se pueden obtener ganancias.

\footnotetext{
2 Trabajo realizado en el marco de una Beca de Estudiante Avanzado otorgada por la Universidad Nacional de Mar del Plata.
} 
Todos los costos fueron re-expresados a valores de marzo 2013, uno de los meses de mayor afluencia de papa del SE bonaerense al mercado. Los valores fueron ajustados mediante el Índice de Precios Internos al por Mayor (IPIM) (Fowler Newton, 2006), publicado por el Instituto Nacional de Estadísticas y Censos (INDEC). Todos los valores están expresados en pesos argentinos.

Se supuso que los productores de papa de la zona son arrendatarios y se contempló sólo la venta en el Mercado Central de Buenos Aires (MCBA) -a través de consignatarios- como canal de comercialización.

Se consideró un rinde de 35 toneladas de papa por hectárea para la Producción Integrada y un precio de venta de $1,51 \$ / \mathrm{kg}$. El mencionado precio se calculó como un $18 \%$ más sobre el precio de venta de la papa Spunta (obtenida bajo producción convencional en el sudeste bonaerense) en marzo de 2013 en el MCBA. El $18 \%$ superior surge de la disponibilidad a pagar (DAP) por una papa con bajo contenido de agroquímicos, manifestada por los consumidores de la ciudad de Mar del Plata en una encuesta realizada en octubre de $2012^{3}$.

Posteriormente, se realizó un "Cash Flow" (calculado por diferencia de ingresos y egresos totales, con una periodicidad anual), es decir, se descontaron -a una tasa $\mathrm{K}$ que representa el costo de oportunidad del capital- los futuros ingresos netos esperados de esta actividad (Bocco y Vence, 2000).

El Flujo de Fondos Descontados tiene las siguientes características:

- Los egresos considerados, fueron estimados en base a los costos de producción calculados en el presente trabajo y se tuvieron en cuenta sólo los gastos efectivos, es decir, no se 
contemplaron las amortizaciones, ya que no son conceptos erogables y por ende, no deben ser tenidas en cuenta al realizar el "Cash Flow".

- Los ingresos se calcularon en base a un rinde promedio de 35 toneladas/ha ${ }^{4}$-suponiendo la implementación de la PI en la zona productiva en estudio- y el precio, considerando la DAP por una papa con menor contenido de agroquímicos, calculada en base a lo manifestado por los consumidores en el relevamiento realizado en octubre de 2012 en la ciudad de Mar del Plata.

- La tasa de descuentos utilizada fue de $9 \%$, sugerida por informantes calificados como una tasa de rentabilidad promedio de una explotación agrícola modal de la zona.

- Horizonte temporal: 3 años, es decir, se consideraron 3 campañas productivas.

- No se consideró la inflación, es decir, no se tuvieron en cuenta las variaciones en el poder adquisitivo de la moneda.

Por último se realizó un análisis de sensibilidad. Dicho análisis consiste en la observación de las variaciones de los criterios de análisis de inversiones ante cambios en alguno de los parámetros que componen el flujo de fondos (Pascale, 2005). Por ello, se plantearon 27 escenarios, modificando rendimientos, precios de venta y la tasa de descuento:

- Rendimientos de papa por hectárea. Se trabajó con 3 niveles de rendimientos: como promedio se utilizó 35 toneladas/ha y a partir de ese valor, se plantearon 2 escenarios: uno pesimista (menor en un $20 \%$ ) y otro optimista (superior en un $20 \%$ ), siendo 28 y 42 toneladas/ha respectivamente.

- Precios de venta. Se utilizó como "precio base" el precio de venta de papa Spunta (proveniente del sudeste bonaerense y obtenida mediante producción convencional) en marzo de

4 Dr. M. Huarte, comunicación personal. 
2013 en el MCBA $(1,28 \$ / \mathrm{kg})^{5}$. Dicha variedad es la de mayor producción y comercialización en el país, ya que, aunque su aptitud culinaria no es la mejor, es con la que se obtienen mayores rendimientos por hectárea. Considerando ese valor, se contemplaron 3 situaciones posibles: que los consumidores no estén dispuestos a pagar un precio mayor para obtener una papa diferenciada (escenario pesimista); que los consumidores paguen un $18 \%$ más para obtener una papa obtenida bajo producción integrada (escenario medio) y que los consumidores estén dispuestos a pagar hasta un $30 \%$ más para adquirir el mencionado producto (escenario optimista), obteniendo finalmente los siguientes precios: $1,28,1,51$ y $1,66 \$ / \mathrm{kg}$.

- Tasa de descuento. Se descontaron los flujos de fondos utilizando 3 tasas de descuento: $6 \%, 9 \%$ y $12 \%$.

\section{Resultados}

\section{Costo de Producción}

A continuación (Tabla 1) se expone el Costo de Producción Integrada de papa en el sudeste bonaerense, calculado en base a un lote de 80 ha, con rendimientos promedio de 35 toneladas/ha y un precio de venta de $1,51 \$ / \mathrm{kg}$ de papa. Los valores están expresados en pesos argentinos ${ }^{6}$.

\footnotetext{
Cabe mencionar que dicho valor se encuentra entre los valores medios (a moneda constante) de los precios pagados en el MCBA por la papa Spunta en marzo de los últimos 5 años. El valor máximo obtenido fue de 2,27 $\$ / \mathrm{kg}$ y el valor mínimo $0,73 \$ / \mathrm{kg}$.

6 Cotización (tipo de cambio vendedor) en el Banco de la Nación Argentina al 31 de marzo de 2013: 1 peso argentino $=0,195$ dólares estadounidenses.
} 
TABLA 1: Costos de producción integrada de papa

\begin{tabular}{|l|c|c|c|c|}
\hline \multicolumn{4}{|c|}{ Producción Integrada } \\
\hline Elemento del Costo & $\begin{array}{c}\text { Costo por Ha } \\
\text { a Marzo 2013 }\end{array}$ & $\begin{array}{c}\text { Costo por } \\
\text { kilo de papa }\end{array}$ & $\begin{array}{c}\text { Costo Total } \\
\text { por 80 Has }\end{array}$ & $\%$ \\
\hline
\end{tabular}

\begin{tabular}{|l|c|c|c|c|}
\hline Arrendamiento & 4229 & 0,12 & 338338 & $8 \%$ \\
\hline Análisis de suelo & 60 & 0,00 & 4770 & $0 \%$ \\
\hline Semilla & 10005 & 0,29 & 800388 & $20 \%$ \\
\hline Monitoreo de plagas & 205 & 0,01 & 16412 & $0 \%$ \\
\hline Sistema de Alarma & 165 & 0,00 & 13237 & $0 \%$ \\
\hline Agroquímicos & 4868 & 0,14 & 389471 & $10 \%$ \\
\hline Fungicidas & 1101 & 0,03 & 88085 & $2 \%$ \\
\hline Labores & 2164 & 0,06 & 173135 & $4 \%$ \\
\hline Riego & 2302 & 0,07 & 184189 & $5 \%$ \\
\hline Cosecha & 6992 & 0,20 & 559344 & $14 \%$ \\
\hline Costos hasta cosecha & $\mathbf{3 2 0 9 2}$ & $\mathbf{0 , 9 2}$ & $\mathbf{2 5 6 7 3 6 9}$ & $\mathbf{6 4 \%}$ \\
\hline Comercialización & 13754 & 0,39 & 1100330 & $27 \%$ \\
\hline $\begin{array}{l}\text { Costos antes de } \\
\text { Depreciaciones }\end{array}$ & $\mathbf{4 5 8 4 6}$ & $\mathbf{1 , 3 1}$ & $\mathbf{3 6 6 7 6 9 9}$ & $\mathbf{9 1 \%}$ \\
\hline Depreciaciones & $\mathbf{4 3 7 5}$ & $\mathbf{0 , 1 3}$ & 350000 & $9 \%$ \\
\hline COSTOS TOTALES & $\mathbf{5 0 2 2 1}$ & $\mathbf{1 , 4 3}$ & $\mathbf{4 0 1 7 6 9 9}$ & $\mathbf{1 0 0 \%}$ \\
\hline
\end{tabular}

FUENTE: Elaboración propia en base a información de informantes calificados.

En la Tabla 2 se muestra el Costo de Producción Convencional de papa en el Sudeste Bonaerense, calculado en base a un lote de 80 ha, con rendimientos promedio de 36,75 toneladas/ha y un precio de venta de 1,28 \$/kg de papa. Los valores están expresados en pesos argentinos. 
TABLA 2: Costos de producción convencional de papa

\begin{tabular}{|l|c|c|c|c|}
\hline \multicolumn{5}{|c|}{ Producción Convencional } \\
\hline Elemento del Costo & $\begin{array}{c}\text { Costo por Ha } \\
\text { a Marzo 2013 }\end{array}$ & $\begin{array}{c}\text { Costo por } \\
\text { kilo de papa }\end{array}$ & $\begin{array}{c}\text { Costo Total } \\
\text { por 80 Has }\end{array}$ & $\%$ \\
\hline
\end{tabular}

\begin{tabular}{|l|c|c|c|c|}
\hline Arrendamiento & 4229 & 0,12 & 338338 & $8 \%$ \\
\hline Semilla & 13723 & 0,37 & 1097877 & $25 \%$ \\
\hline Agroquímicos & 4868 & 0,13 & 389471 & $9 \%$ \\
\hline Fungicidas & 1268 & 0,03 & 101475 & $2 \%$ \\
\hline Labores & 2231 & 0,06 & 178496 & $4 \%$ \\
\hline Riego & 2302 & 0,06 & 184189 & $4 \%$ \\
\hline Cosecha & $\mathbf{7 3 3 8}$ & 0,20 & 587049 & $14 \%$ \\
\hline Costos hasta cosecha & $\mathbf{3 5 9 6 1}$ & $\mathbf{0 , 9 8}$ & $\mathbf{2 8 7 6 8 9 5}$ & $\mathbf{6 6 \%}$ \\
\hline Comercialización & 13759 & 0,37 & 1100737 & $25 \%$ \\
\hline $\begin{array}{l}\text { Costos antes de } \\
\text { Depreciaciones }\end{array}$ & $\mathbf{4 9 7 2 0}$ & $\mathbf{1 , 3 5}$ & $\mathbf{3 9 7 7 6 3 1}$ & $\mathbf{9 2 \%}$ \\
\hline Depreciaciones & $\mathbf{4 3 7 5}$ & $\mathbf{0 , 1 2}$ & 350000 & $8 \%$ \\
\hline COSTOS TOTALES & $\mathbf{5 4 0 9 5}$ & $\mathbf{1 , 4 7}$ & $\mathbf{4 3 2 7 6 3 1}$ & $\mathbf{1 0 0 \%}$ \\
\hline
\end{tabular}

FUENTE: Elaboración propia en base a información de informantes calificados.

En la producción convencional, se estimó un rendimiento $5 \%$ mayor al obtenido bajo producción integrada (1312 bolsas versus 1250 bolsas, respectivamente) ya que con las variedades utilizadas en la producción convencional se obtienen mayores rindes por hectárea ${ }^{7}$. Para estimar el costo de comercialización, se utilizó el precio de venta de la papa Spunta en el MCBA en marzo de 2013.

7 El Protocolo recomienda emplear en la Producción Integrada cultivares resistentes o tolerantes a las principales plagas y enfermedades de la zona, por ejemplo: Frital INTA, Pampeana INTA, Calen INTA, Newen, entre otras. 
En las Tablas 1 y 2 se pueden observar rubros de costos que son comunes en la estructura de costos de ambas producciones de papa -arrendamiento, semilla, agroquímicos, fungicidas, labores, riego, cosecha, comercialización y depreciaciones- pero que, algunos, difieren en su cuantía y "costos nuevos", propios de la producción integrada -análisis de suelo, monitoreo de plagas y sistema de alarma de Phytophthora.

Teniendo en cuenta el costo de producción por kilo de papa obtenido bajo PI (1,43 \$) y el precio, en la situación pesimista (DAP 1,28 \$/kg) representaría un perjuicio económico. Sin embargo, el costo de producción resulta levemente menor a la DAP, considerando el caso del escenario medio (DAP 1,51 \$/kg) y del escenario optimista (DAP 1,66 \$/kg), para una papa con menor contenido de agroquímicos.

En la Tabla 2 puede observarse que la diferencia entre el costo de producción por kilo de papa obtenido bajo producción convencional y el precio realmente pagado por ella -en el mismo momento y lugar-, es ligeramente mayor.

La diferencia entre el costo total de producción de ambos sistemas productivos se debe principalmente a:

Menor costo de la papa semilla. En la campaña analizada puede observarse un costo significativamente menor de la semilla utilizada en la PI en relación a la utilizada en la PC, caáusado principalmente por la diferencia de variedades utilizadas por cada sistema productivo. El costo de la papa semilla está asociado al de la papa consumo, por lo cual puede ocurrir que en otra campaña productiva, el costo de semilla sea mayor en la PC que en la PI. La cantidad de semilla utilizada en la PI puede llegar a ser hasta un $15 \%$ menor a la utilizada en la PC en caso de sembrar variedades como Pampena INTA, Newen o Frital INTA. Esto no se contempló en 
nuestras estimaciones, ya que la cantidad utilizada de papa semilla fue la misma para ambos sistemas productivos.

Ahorro de fungicidas ocasionado por la utilización del Sistema de Alarma de Phytophthora-, no sólo en el costo de este tipo de agroquímico sino en el costo que implica su aplicación (este último ahorro puede visualizarse en el rubro "Labores"). Cabe destacar que el ahorro en fungicidas variará campaña a campaña dependiendo básicamente de las condiciones ambientales para la infección con Phytophthora; en este caso en particular puede apreciarse una ahorro de alrededor del $13 \%{ }^{8}$ pero se estima que pueden producirse ahorros de hasta el $20 \%$.

La implementación del Sistema de Alarma, el análisis de suelos y el monitoreo de plagas, aunque son costos propios de la PI, son insignificantes en relación a otros elementos del costo.

El costo de cosecha es menor en la Producción Integrada debido al menor rendimiento por hectárea.

Con respecto al costo de comercialización se da una situación especial ya que depende de 2 variables: el rendimiento y el precio de venta. En la Producción Integrada los mayores precios a lograr por este producto, se contrarrestan con el menor rendimiento por hectárea, en relación a los obtenidos por la convencional. En definitiva, el costo de comercialización por hectárea es similar en ambos sistemas productivos.

\footnotetext{
Egresos e Ingresos bajo distintos escenarios de precios y rendimientos. Producción Integrada.

8 El mencionado valor surge de comparar las aplicaciones realmente realizadas en un lote durante una campaña productiva (implementando el Sistema de Alarma) y las supuestas aplicaciones realizadas sin el Sistema de Alarma, es decir, realizando aplicaciones una vez a la semana.
} 
Con el fin de calcular los diferentes egresos para los 27 escenarios, se modificaron 2 variables precio y rendimiento- que influyeron en los costos de cosecha y comercialización. Es decir, se modificaron sólo los costos de cosecha y comercialización, permaneciendo fijos los demás elementos del costo, ya que son independientes de las variables mencionadas. Al tratarse solamente de conceptos erogables, no se tuvieron en cuenta las depreciaciones.

En la Tabla 3 se exponen los costos erogables estimados para una Producción Integrada de 80 ha de papa, teniendo en cuenta los precios de bienes y servicios de la campaña 2012-2013 y contemplando 3 niveles de rendimientos y de precios de venta.

TABLA 3: Costos erogables totales (pesos) de la Producción Integrada

\begin{tabular}{|c|c|c|c|c|}
\cline { 3 - 5 } \multicolumn{2}{c|}{} & \multicolumn{3}{c|}{ Rendimiento (t/ha ) } \\
\cline { 3 - 5 } \multicolumn{2}{c|}{} & 28 & 35 & 42 \\
\hline \multirow{3}{*}{ Precios $(\$ / \mathrm{kg})$} & 1,28 & 3294631 & 3616089 & 3937548 \\
\cline { 2 - 5 } & 1,51 & 3335919 & 3667699 & 3999479 \\
\cline { 2 - 5 } & 1,66 & 3363444 & 3702105 & 4040767 \\
\hline
\end{tabular}

FUENTE: Elaboración propia en base a información de informantes calificados.

Como puede apreciarse, los menores costos se registran a menores rendimientos por hectárea.

$\mathrm{Al}$ aumentar los rendimientos, aumentan los costos de cosecha y comercialización.

En la Tabla 4 se exponen los ingresos estimados para la Producción Integrada de 80 ha de papa, a 3 niveles de precios y rendimientos.

TABLA 4 : Ingresos totales (pesos) en la Producción Integrada

\begin{tabular}{|c|c|c|c|c|}
\hline & \multicolumn{3}{|c|}{ Rendimiento ( $\mathrm{t} / \mathrm{ha}$ ) } \\
\hline & & 28 & 35 & 42 \\
\hline \multirow{3}{*}{ Precios $(\$ / \mathrm{kg})$} & 1,28 & 2867200 & 3584000 & 4300800 \\
\hline & 1,51 & 3383200 & 4229000 & 5074800 \\
\hline & 1,66 & 3727200 & 4659000 & 5590800 \\
\hline
\end{tabular}

FUENTE: Elaboración propia en base a información de informantes calificados. 


\section{Análisis de escenarios}

En la Tabla 5 se plantean 27 escenarios con los beneficios netos calculados en base al "Cash Flow" descontado a tasas del 6\%,9\% y $12 \%$ anual, para 3 campañas productivas bajo el sistema de PI.

Es oportuno aclarar que al no existir datos históricos de rendimientos ni precios de papa obtenida bajo PI, ya que es una modalidad productiva que sólo ha sido realizada experimentalmente, no puede ponderarse el riesgo de ocurrencia de los beneficios netos negativos en base a situaciones históricas más frecuentes.

Como puede observarse, existen 6 situaciones en las que los resultados obtenidos serían negativos; todos ellos surgen de un escenario pesimista: suponiendo que los consumidores no estarían dispuestos a pagar un valor adicional para adquirir una papa con menor contenido de agroquímicos y que sólo pagarían el mismo valor que una papa obtenida bajo producción convencional $(1,28 \$ / \mathrm{kg})$.

El mejor resultado surge de la combinación de un precio de venta de 1,66 \$/kg de papa (suponiendo una DAP superior en un $30 \%$ al precio de venta de la papa Spunta en el MCBA), rendimiento de 42 t/ha y una tasa de descuento del $6 \%$. Si mantenemos el mismo precio y rendimiento, pero descontamos los flujos a un $12 \%$, también se obtendría un resultado más que aceptable. 
TABLA 5: Beneficio neto estimado en pesos para la Producción Integrada con 3 niveles de precios, rendimientos y tasas de descuento.

\begin{tabular}{|c|c|c|c|c|c|c|c|c|c|c|}
\hline & \multicolumn{9}{|c|}{ Tasa de descuento (\%) y Rendimientos de papa (t/ha) } \\
\hline & & \multicolumn{3}{|c|}{$6 \%$} & \multicolumn{3}{|c|}{$9 \%$} & \multicolumn{3}{|c|}{$12 \%$} \\
\hline & & 28 & 35 & 42 & 28 & 35 & 42 & 28 & 35 & 42 \\
\hline \multirow{3}{*}{$\begin{array}{c}\text { Precios } \\
(\$ / \mathrm{kg})\end{array}$} & 1,28 & -1142529 & -85775 & 970978 & -1081954 & -81228 & 919499 & -1026618 & -77073 & 872471 \\
\hline & 1,51 & 126383 & 1500364 & 2874346 & 119683 & 1420818 & 2721954 & 113561 & 1348150 & 2582739 \\
\hline & 1,66 & 972324 & 2557791 & 4143257 & 920774 & 2422182 & 3923591 & 873681 & 2298300 & 3722918 \\
\hline
\end{tabular}

FUENTE: Elaboración propia en base a información de informantes calificados.

\section{Consideraciones finales}

La papa tiene un alto costo de producción que es difícil de disminuir sin afectar los rendimientos, no obstante, implementando un sistema de Producción Integrada, utilizando al máximo los recursos y mecanismos de regulación naturales, es posible disminuir el uso de agroquímicos y riego obteniendo un producto de mayor inocuidad y calidad a un costo similar o incluso inferior al correspondiente a la producción convencional de papa y por ende, disminuir sus egresos.

Por otra parte, los ingresos dependerán de los rendimientos y de los precios de venta del producto. Ante rendimientos típicos de la zona en estudio, los resultados dependen de los precios de venta.

De no existir diferencia entre el precio obtenido por la papa bajo una modalidad u otra, la pequeña diferencia en los costos de producción (menor al $5 \%$ ) probablemente no sea un incentivo suficiente para que los productores adopten la PI, ya que involucra inversiones intangibles tales como adquirir nuevos conocimientos sobre las prácticas productivas, realizar un mayor seguimiento del cultivo, entre otras. La obtención de un precio superior, equivalente a la DAP del $18 \%$, que surgió del estudio realizado con consumidores, sí podría significar un importante estímulo para la implementación de la PI. Para ello, los productores de papa y los agentes que intervienen en la cadena de comercialización tendrían que centrar su atención en la 
diferenciación del producto y, mediante una marca o sello de calidad que permita al consumidor identificarlo y reconocer las diferencias, sería factible lograr este mayor precio que los consumidores estarían dispuestos a pagar.

\section{Conflictos de interés}

Los autores declaran que no existen conflictos de interés.

\section{Agradecimientos}

Al Dr. Marcelo A. Huarte por los valiosos comentarios realizados a una versión preliminar de este trabajo.

Trabajo realizado en el marco del Proyecto "Medición de atributos de calidad y preferencias por productos obtenidos con bajo impacto ambiental”. Facultad de Ciencias Económicas y Sociales. Universidad Nacional de Mar del Plata. Argentina

\section{Literatura citada}

Bocco G.L.; Vence L.A. 2000. Capítulo 4: Métodos de Evaluación de Proyectos de Inversión. Pp 45-49. En: Proyectos de Inversión. Métodos de Evaluación. Problemas y Aspectos Especiales. Editorial Errepar. Buenos Aires. Argentina.

Fowler Newton E. 2006. "Contabilidad Superior", 5ta edición, Editorial La Ley, Buenos Aires, Argentina.663 p.

González, M.C. y Pagliettini, L.L. 2001. Los Costos Agrarios y sus aplicaciones. Editorial Facultad de Agronomía, Universidad de Buenos Aires, Argentina. 75 p.

Huarte, M; Huarte D.; Lucca, F; Carmona, D.; Mairosser, A.; Viglianchino, L. 2011. Protocolo para la Producción Integrada de papa consumo en el sudeste de la provincia de Buenos Aires. Primera Edición. Ediciones INTA Publicaciones Regionales. 25 p.

Lupín, B, Rodriguez E. M. M. y González, J. 2013. Evaluación de preferencias de atributos aplicando Choice Modelling. Reunión Anual de la Asociación Argentina de Economía Agraria (AAEA). San Juan, Argentina. 935 p. 
Pascale R. 2005. Decisiones Financieras. Cuarta Edición. Fondo Editorial Consejo de Ciencias Económicas de la Ciudad Autónoma de Buenos Aires. Ciudad Autónoma de Buenos Aires. 810 p.

Remondino, H.D. y Garino A.T. 2003. Medición y Evaluación de Resultados de Empresas Agropecuarias. Revista Enfoques. Argentina.

Rodríguez, J. A. y Rodríguez, E. M. M. 2013. Producción Integrada de papa versus Producción Convencional: Costos de Producción y Flujo de Fondos en el sudeste de la provincia de Buenos Aires. Reunión Anual de la Asociación Argentina de Economía Agraria (AAEA). San Juan, Argentina. $935 \mathrm{p}$. 